\title{
Deep Learning-Based Energy Disaggregation and On/Off Detection of Household Appliances
}

\author{
JIE JIANG, QIUQIANG KONG, MARK D. PLUMBLEY, and NIGEL GILBERT, \\ University of Surrey \\ MARK HOOGENDOORN and DIEDERIK M. ROIJERS, Vrije Universiteit Amsterdam
}

\begin{abstract}
Energy disaggregation, a.k.a. Non-Intrusive Load Monitoring, aims to separate the energy consumption of individual appliances from the readings of a mains power meter measuring the total energy consumption of, e.g., a whole house. Energy consumption of individual appliances can be useful in many applications, e.g., providing appliance-level feedback to the end users to help them understand their energy consumption and ultimately save energy. Recently, with the availability of large-scale energy consumption datasets, various neural network models such as convolutional neural networks and recurrent neural networks have been investigated to solve the energy disaggregation problem. Neural network models can learn complex patterns from large amounts of data and have been shown to outperform the traditional machine learning methods such as variants of hidden Markov models. However, current neural network methods for energy disaggregation are either computational expensive or are not capable of handling long-term dependencies. In this article, we investigate the application of the recently developed WaveNet models for the task of energy disaggregation. Based on a real-world energy dataset collected from 20 households over 2 years, we show that WaveNet models outperforms the state-of-the-art deep learning methods proposed in the literature for energy disaggregation in terms of both error measures and computational cost. On the basis of energy disaggregation, we then investigate the performance of two deep-learning based frameworks for the task of on/off detection which aims at estimating whether an appliance is in operation or not. The first framework obtains the on/off states of an appliance by binarising the predictions of a regression model trained for energy disaggregation, while the second framework obtains the on/off states of an appliance by directly training a binary classifier with binarised energy readings of the appliance serving as the target values. Based on the same dataset, we show that for the task of on/off detection the second framework, i.e., directly training a binary classifier, achieves better performance in terms of F1 score.
\end{abstract}

CCS Concepts: $\bullet$ Computing methodologies $\rightarrow$ Neural networks; $\bullet$ Theory of computation $\rightarrow$ Structured prediction;

Additional Key Words and Phrases: Energy disaggregation, non-intrusive load monitoring, deep learning

This work was carried out as part of the "HomeSense: digital sensors for social research" project funded by the Economic and Social Research Council (grant ES/N011589/1) through the National Centre for Research Methods. Qiuqiang Kong was supported by EPSRC grant EP/N014111/1 "Making Sense of Sounds" and a Research Scholarship from the China Scholarship Council (CSC) No. 201406150082.

Current Authors' address: Diederik M. Roijers's current affiliation is Vrije Universiteit Brussel, Artificial Intelligence Lab Brussels, Elsene, 1050, Belgium, and HU University of Applied Sciences Utrecht, Netherlands.

Authors' addresses: J. Jiang, Q. Kong (corresponding author), M. D. Plumbley, and N. Gilbert, University of Surrey, Guildford, Surrey, GU2 7XH, United Kingdom; emails: \{jie.jiang, q.kong, m.plumbley, n.gilbert\}@surrey.ac.uk; M. Hoogendoorn and D. M. Roijers, Vrije Universiteit Amsterdam, Amsterdam, 1081 HV, Netherlands; emails: m.hoogendoorn@vu.nl, diederik.roijers@vub.be.

This work is licensed under a Creative Commons Attribution-Share Alike International 4.0 License.

(c) 2021 Copyright held by the owner/author(s).

1556-4681/2021/04-ART50

https://doi.org/10.1145/3441300 


\section{ACM Reference format:}

Jie Jiang, Qiuqiang Kong, Mark D. Plumbley, Nigel Gilbert, Mark Hoogendoorn, and Diederik M. Roijers. 2021. Deep Learning-Based Energy Disaggregation and On/Off Detection of Household Appliances. ACM Trans. Knowl. Discov. Data 15, 3, Article 50 (April 2021), 21 pages.

https://doi.org/10.1145/3441300

\section{INTRODUCTION}

In recent years, several large-scale household energy consumption datasets are made publicly available, e.g., UK-DALE [24] (UK Domestic Appliance-Level Electricity) and REFIT [34] (Personalised Retrofit Decision Support Tools For UK Homes Using Smart Home Technology). These datasets boost the studies on energy disaggregation, also known as Non-Intrusive Load Monitoring (NILM) [18]. Energy disaggregation is a challenging blind source separation problem that aims to separate the energy consumption of individual appliances from the readings of the aggregate meter measuring the total consumption of multiple appliances for example in a house. Figure 1 gives an example of how the energy consumption of a whole house changes along with that of the individual appliances. This problem is difficult due to a number of uncertainties such as the existence of background noise, the lack of knowledge on the numbers of different appliances and their true energy consumption patterns in a given household, replacements of old appliances, and overlapped operations of multiple appliances with similar energy consumption patterns.

Energy disaggregation finds its usefulness in many applications. For example, disaggregated data could be used by feedback systems to provide pertinent information about energy usage and educate consumers at opportune times [14], which in turn helps the consumers better control their consumption and ultimately save energy [13]. Disaggregated data may also help identify malfunctioning equipments or inefficient settings [15]. For policymakers, knowing the amount of energy each category of appliances consumes is critical to the development and evaluation of energy efficiency policies [40,41]. Disaggregated data may also provide valuable information to facilitate power system planning, load forecasting, new types of billing procedures, and the ability to pinpoint the origins of certain customer complaints [15]. Another application is to help researchers understand the occurrences of home activities which nowadays are heavily related with the usage of different types of appliances [22].

In the literature, a lot of research has been done on applying machine learning methods to the problem of energy disaggregation. Popular approaches include different variants of Hidden Markov Models (HMMs) such as Factorial HMMs (FHMMs) (e.g., [29, 38, 52]), sparse coding (e.g., [12, 25, 28]), graph signal processing (GSP) (e.g., [19, 50, 51]) and integer programming (IP) (e.g., [7, 11, 30]). Among these approaches, HMM-based and GSP-based energy disaggregation methods have been explored under both supervised and unsupervised settings. Sparse coding based methods mostly adopt a supervised setting while IP based methods do not require any training. With the availability of large-scale open datasets such as UK-DALE and REFIT [24, 34], there has been a focus on applying deep neural networks (DNNs) to the problem of energy disaggregation. For example, in [23] and [49], the authors investigated the application of convolutional neural networks (CNNs), recurrent neural networks (RNNs) and Autoencoders, and show that the DNNs-based energy disaggregation methods outperform those based on traditional methods such as FHMMs. However, there are several problems with the conventional DNN models. The computation complexity of the conventional CNNs gets substantially higher when the input sequences are long. In the case of RNNs, the values of the hidden units have to be calculated in a sequential order and thus does not scale well. Recently, a neural network called WaveNet [46] was proposed for long sequence audio processing. WaveNet is a variant of the $\mathrm{CNN}$ architecture with dilated convolutional layers which make it easier to be trained with long sequences compared 


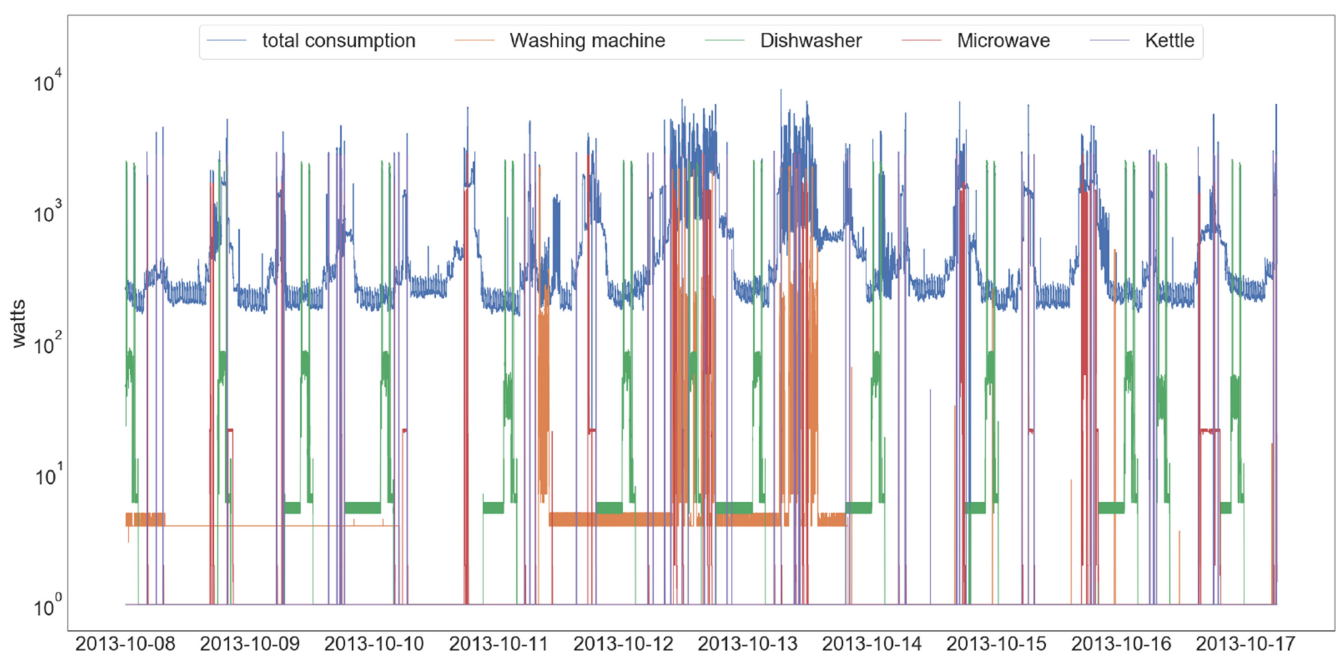

Fig. 1. An example of energy consumption of individual appliances and a whole house.

to the conventional CNNs. With skip connections over all the convolutional layers, it can learn multi-scale hierarchical representations. WaveNet has been proven to work well for tasks such as speech synthesis [46] and speech denoising [37]. It is efficient because it has fewer parameters than a CNN does. WaveNet is also easy to parallelise compared to RNNs. For the task of energy disaggregation, some appliances may have long-term dependencies in their energy consumption patterns and these patterns may exist at different scales. Therefore, the task of energy disaggregation may benefit from WaveNet's capability of modeling long sequences and learning multi-scale hierarchical representations.

To evaluate the performance of WaveNet models for the task of energy disaggregation, we carried out a set of experiments using the public dataset REFIT [34], and compared the disaggregation results of WaveNet models against the five-layer CNN model proposed in [49], the two-layer bidirectional RNN model with long short-term memory (LSTM) units proposed in [23] and a threelayer bidirectional RNN model with gated recurrent units (GRUs). We showed that WaveNet models outperform the other three methods in terms of both error measures and computation cost. We also investigated the influence of the length of input sequences on the disaggregation performance as well as on the computation cost.

While the problem of energy disaggregation focuses on estimating the exact amount of energy being consumed by an appliance, there is another interesting task called on/off detection which tries to predict whether individual appliances are in operation or not. Compared to energy disaggregation, on/off detection provides a perspective of a coarser granularity on the usage status of individual appliances and finds its usefulness in understanding the occurrences of home activities that heavily depend upon the assistance of household appliances such as kettle [3], washing machine and microwave [22]. Such dependencies have been proven, for example, to help with activity monitoring and health management [3]. In this article, we investigate two learning frameworks for the task of on/off detection. The first one, called the regression based learning framework, first trains a model for energy disaggregation using the aggregate energy readings as inputs and the appliance readings as the target values, and then derives the on/off state sequence of the appliance by binarising the predictions of the disaggregation model according to the on-power threshold of the appliance. The second one, called the classification based learning framework, directly trains a binary classifier with the appliance on/off states. 
Table 1. Notation Used in This Article

\begin{tabular}{|c|c|}
\hline Notation & Explanation \\
\hline$x_{t}$ & house-level energy reading at time $t$ \\
\hline$X$ & a sequence of house-level energy readings \\
\hline$y_{t}^{i}$ & energy reading of an appliance $i$ at time $t$ \\
\hline$Y^{i}$ & a sequence of energy readings of appliance $i$ \\
\hline$u_{t}$ & reading of unknown appliances and background noise at time $t$ \\
\hline$z_{t}^{i}$ & on/off state of an appliance $i$ at time $t$ \\
\hline$f^{i}$ & $\begin{array}{l}\text { a mapping from a sequence of house-level energy readings to a sequence of energy readings or on/off } \\
\text { states of appliance } i\end{array}$ \\
\hline$L$ & receptive field size \\
\hline$r$ & target field size \\
\hline$s$ & number of dilated convolutional layers \\
\hline$m$ & filter length applied to each dilated convolutional layer \\
\hline$\hat{\boldsymbol{y}}_{t}$ & predicted appliance energy reading at time $t$ \\
\hline$\hat{z}_{t}$ & predicted appliance energy on/off state at time $t$ \\
\hline$a$ & energy consumption of an appliance over a certain time period \\
\hline$\hat{a}$ & predicted energy consumption of an appliance over a certain time period \\
\hline
\end{tabular}

To evaluate the two learning frameworks for the task of on/off detection, we respectively trained a group of WaveNet models following the two learning frameworks with the REFIT dataset. We showed that for the task of on/off detection the classification based learning framework outperforms the regression based learning framework in terms of F1 score.

The contributions of this article are as follows:

-We propose to tackle the problem of energy diaggregation with WaveNet models which are capable of modeling long sequences more efficiently compared to the conventional CNNs and RNNs, and we show that WaveNet models achieve the state-of-the-art performance based on a set of experiments with a public dataset.

-We carry out an analysis on how the receptive field size and the target field size would affect the disaggregation performance of the three DNNs, i.e., WaveNets, CNNs and RNNs.

-We compare a regression-based learning framework with a classification-based learning framework for the task of on/off detection and show empirically that the latter outperforms the former that uses the outputs from energy disaggregation.

- The evaluation is performed using the public dataset REFIT collected from 20 households. We give a detailed description of how the raw data was preprocessed and used for model training and release the source $\operatorname{code}^{1}$ to facilitate the reproducibility of our work.

The rest of the article is organized as follows: Section 2 discusses the related work. Section 3 gives a formal description of the energy disaggregation problem and the on/off detection problem. Section 4 presents three learning paradigms for model training, introduces three neural network models and describes how a model is trained respectively for the task of energy disaggregation and on/off detection. Section 5 illustrates the experiment preparation and analyses the experiment results. Finally, in Section 6, we conclude the article with possibilities of future work.

The notation used in this article is summarised in Table 1.

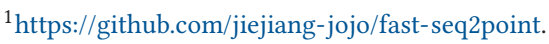




\section{RELATED WORK}

In the literature, a lot of research has been done on applying machine learning methods to the problem of energy disaggregation. Among the popular approaches, different variants of HMMs, in particular the FHMMs, have attracted much attention (e.g., [26, 29, 35, 38, 52]). These HMM-based approaches work in a supervised setting (e.g., [38, 52]) or an unsupervised setting (e.g., [26, 29, 35]). However, since their learning process often relies on the expectation-maximization algorithm which depends on initialisation, they may achieve local-optima solutions. Another widely studied method for the problem of energy disaggregation is sparse coding (e.g., [12, 25, 28, 42]) because it brings an additional constraint of sparse activations while providing a natural formulation of a single-channel source separation problem. Most of the sparse coding-based approaches adopted a supervised setting. More recently, researchers extended sparse coding to deep structures [42] and integrated it with other deep learning methods such as LSTM and autoencoder [25]. In both HMM and sparse coding-based energy disaggregation, different priors have been investigated to improve the disaggregation performance such as time of day, and knowledge about devices that do or do not work together. GSP-based energy disaggregation approaches are also being actively explored under both supervised [19] and unsupervised settings [51]. To mitigate measurement noise and the influence of unknown appliances, [50] exploits GSP's properties as effective physical signal filters. There is also research applying IP techniques to solve the problem of energy disaggregation (e.g., $[7,11,30])$ by determining which appliance states are active at any given time. Though such methods do not require any training, a lot of parameters have to be determined separately such as the number of states for each appliance as well as their steady-state ratings [7].

Recently, with the availability of large open datasets such as UK-DALE and REFIT [24, 34] and the superior performance of DNNs in many research areas such as computer vision [31] and audio processing [37], there has been a focus on applying DNNs for the problem of energy disaggregation. For example, Kelly and Knottenbelt [23] compared the disaggregation performance of the traditional machine learning methods (e.g., FHMMs) with the deep learning methods such as $\mathrm{Au}-$ toencoders and LSTM networks and the results show that the deep learning methods outperform the traditional methods. Mauch and Yang [33] also advocated the application of LSTM for the problem of energy disaggregation. Chen et al. [8] proposed a convolutional sequence to sequence model in which gated linear unit convolutional layers were used to extract information from the sequences of aggregate electricity consumption and residual blocks were used to refine the output of the neural network. Later, Zhang et al. [49] proposed to use a sequence-to-point paradigm to train a $\mathrm{CNN}$ for energy disaggregation which outperforms the sequence-to-sequence learning approach used in [23]. There are also works using a combination of DNNs. For example, by combining CNNs with variational autoencoders, Sirojan et al. [44] showed that their approach outperforms the one presented in [49]. Shin et al. [39] proposed a subtask gated network that combines the main regression network with an on/off classification subtask network. Targeting real-time applications, Harell et al. [17] proposed a causal 1-D CNN based on the WaveNet model proposed in [46]. This work is similar to ours as it also adapts WaveNet for the problem of energy disaggregation but our work differs from this work as we use a non-causal version of WaveNet proposed in [37], i.e., the same amount of samples in the past as well as in the future are used to train the model and inform the prediction. Another difference is that we employed the concept of target field as proposed in [37] such that the computation of the neighbouring samples can be shared, which speeds up the model training and inference. In addition, we carried out an extensive study on how the receptive field size and the target field size influence the model performance. Moreover, the baseline used in [17] is a variant of HMM, i.e., sparse super-state HMM, while we compared our work with the state-of-the-art DNN based approaches. 
Another group of works that are relevant to this study are those taking NILM as a multi-label classification problem (e.g., [4, 5, 32, 43, 45, 47]). These works make use of annotated aggregated load for model training, which contains information on the on/off state of the target devices. In contrast to binary label classification where one sample belongs to only one output class, multilabel classification allows a sample being mapped to multiple output classes (appliances). An early study investigating multi-label classification methods for NILM was presented in [5], which provided a comparison of several multi-label classification algorithms such as multi-label K nearest neighbour and random $\mathrm{K}$ label-sets. More recent works are based on multi-label deep learning [43], multi-label graph learning [32], multi-label restricted Boltzmann machine [47]. In this work, multi-label classification is realised by training a model for each appliance to identify if the appliance is in an on/off state. Therefore, multiple appliances may be recognised as in an on state at the same time instance. In particular, this work investigates the application of the state-of-the-art deep learning methods for the task of appliance on/off detection.

\section{PROBLEM STATEMENT}

\subsection{Energy Disaggregation}

Energy disaggregation aims to estimate the energy usage of individual appliances based on the readings of the mains power meter that measures the total energy consumption of, for example, a whole house. Formally, suppose we have a sequence of readings from a house-level meter denoted as $X=\left(x_{1}, x_{2}, \ldots, x_{T}\right)$ where $T$ is the length of the sequence. The problem of energy disaggregation is to disaggregate $X$ into the energy consumption sequence of individual appliances denoted as $Y^{i}=\left(y_{1}^{i}, y_{2}^{i}, \ldots, y_{T}^{i}\right), y_{t}^{i} \in \mathbb{R}_{\geq 0}$ where $\mathbb{R}_{\geq 0}=[0,+\infty), I$ is the number of known appliances, $i \in\{1, \ldots, I\}$ is the appliance index and $t \in\{1, \ldots, T\}$ is the index of samples in time domain. In addition, we denote the readings from unknown appliances and background noise as $U=\left(u_{1}, \ldots, u_{T}\right)$. At any time $t, x_{t}$ is assumed to be the summation of the readings from all the known appliances and unknown appliances with background noise:

$$
x_{t}=\sum_{i=1}^{I} y_{t}^{i}+u_{t},
$$

where the residual term $u_{t}$ indicates the energy consumption of unknown appliances and background noise at time $t$. The aim of energy disaggregation is to design a model to separate the energy consumption of the individual appliances $Y^{i}, i \in\{1, \ldots, I\}$ from the aggregate readings $X$. That is, we are looking for a set of disaggregation mappings:

$$
f^{i}: X \mapsto Y^{i},
$$

where each mapping $f^{i}$ maps from an aggregate reading sequence $X$ to the energy consumption sequence of an appliance $Y^{i}$.

\subsection{On/off Detection}

On a coarser granularity, most appliances have an on-power threshold which defines the least amount of energy an appliance needs to operate. When the amount of energy an appliance is consuming is below the on-power threshold it is considered to be in an off state otherwise it is considered to be in an on state. For example, a kettle usually needs 2,000 watts to be in an on state while a washing machine needs 20 watts. The problem of on/off detection aims to estimate whether an appliance is in an on or off state based on the readings of the total energy consumption.

Formally, given a sequence of aggregate readings from a house-level meter denoted as $X=\left(x_{1}, x_{2}, \ldots, x_{T}\right)$ where $T$ is the length of the sequence, the aim of on/off detection is to 


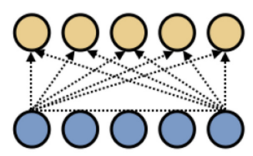

(a)

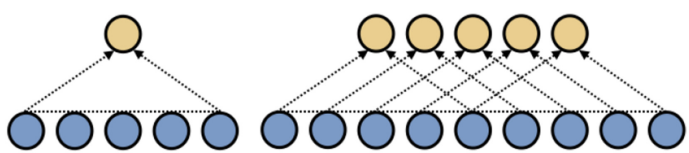

(c)

Fig. 2. (a) Sequence-to-sequence learning; (b) Sequence-to-point learning; (c) Fast sequence-to-point learning.

design a model to recognise the on/off state sequence of individual appliances denoted as $Z^{i}=$ $\left(z_{1}^{i}, z_{2}^{i}, \ldots, z_{T}^{i}\right), z_{t}^{i} \in\{0,1\}, i \in\{1, \ldots, I\}, t \in\{1, \ldots, T\}$ from the aggregate readings $X$ where $I$ is the number of known appliances, $i$ is the appliance index, $t$ is the sample index in time domain, 0 indicates the off state and 1 indicates the on state. That is, we are looking for a set of mappings:

$$
f^{i}: X \mapsto Z^{i}
$$

where each mapping $f^{i}$ maps from an aggregate reading sequence $X$ to the on/off state sequence of an appliance $Z^{i}$.

\section{METHODS}

\subsection{Learning Paradigms}

Usually the aggregate readings $X$ is a long sequence over days, months, or sometimes years. To efficiently train a model, a commonly used approach in the literature of energy disaggregation is the sliding window approach which splits the long sequence $X$ into shorter sequences $\mathbf{x}=\left(x_{t}, \ldots, x_{t+L-1}\right)$ where $L$ indicates the receptive field size. Instead of learning the mappings in Equations (2) and (3) directly, we use sequences $\mathbf{x}$ as input. The target of an input sequence can be a sequence of the same length which is called sequence-to-sequence learning [23] or the midpoint of the target sequence which is called sequence-to-point learning [49]. In this section, we introduce three variants of the sliding window approach.

4.1.1 Sequence-to-sequence Learning. Sequence-to-sequence learning [23], as shown in Figure 2(a), was proposed to learn a mapping from an input sequence $\mathbf{x}$ to an output/target sequence $\mathbf{y}$, where $\mathbf{x}=\left(x_{t}, \ldots, x_{t+L-1}\right)$ and $\mathbf{y}=\left(y_{t}, \ldots, y_{t+L-1}\right)$ have the same length. In sequenceto-sequence learning, each element of the output signal is predicted many times and an average of these predictions is used as the final output, which consequently smooths the edges. However, as pointed in [49], it is expected that some of the input sequences will provide a better prediction of a single point than others, particularly those sequences where the point is near the midpoint of the input sequence.

4.1.2 Sequence-to-point Learning. Sequence-to-point learning [49], as shown in Figure 2(b), aims to solve the problem of sequence-to-sequence learning by finding a mapping from an input sequence $\mathbf{x}=\left(x_{t}, \ldots, x_{t+L-1}\right)$ to a single target point $y_{t+\lfloor L / 2\rfloor}$ the index of which corresponds to the midpoint of the input sequence, where $\lfloor\cdot\rfloor$ floors a value to an integer. One problem of the sequence-to-point learning paradigm is that learning a single point is usually inefficient.

4.1.3 Fast Sequence-to-point Learning. In this article, we propose to use a fast sequence-to-point learning paradigm, as shown in Figure 2(c), to speed up the sequence-to-point learning. By introducing a target field [37] to replace a single point as output, the computation of a sequence-to-point model can be shared. The input sequence and target sequence are denoted as $\mathbf{x}=\left(x_{t}, \ldots, x_{t+L+r-2}\right)$ and $\mathbf{y}=\left(y_{t+\lfloor L / 2\rfloor}, \ldots, y_{t+\lfloor L / 2\rfloor+r-1}\right)$, respectively. The length of the input sequence in this case is $(L+r-1)$ where $L$ indicates the size of the receptive field, and $r$ indicates the size of the target 


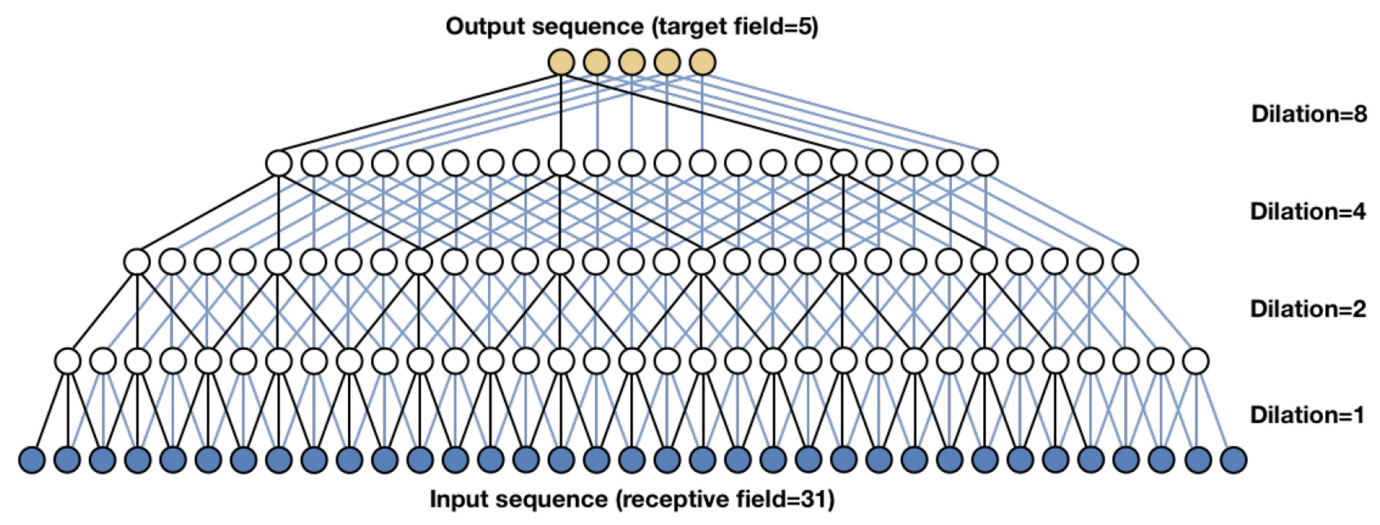

Fig. 3. An example of the WaveNet input-output structure for energy disaggregation.

field, i.e., the length of the target sequence. When $r=1$, fast sequence-to-point learning degenerates to sequence-to-point learning.

\subsection{WaveNets}

CNNs have achieved the state-of-the-art performance in many applications such as computer vision [31], speech and audio processing [37] and natural language processing [10]. With shared filters to capture local patterns of various signals, the number of parameters of a CNN is fewer than that of a fully connected neural network.

Another type of DNN models, RNNs, have many successful applications in modeling temporal signals, e.g., audio and speech signal processing [16] and natural language processing [9]. With connections between adjacent hidden units in RNNs, temporal dynamic behaviour can be captured. In a non-causal system, a RNN can be bidirectional so as to use information from both history and future. To solve the problem of gradient vanishing/explosion [36], LSTM was proposed [20] and later GRU [9] was proposed to simplify LSTM by reducing the number of parameters.

However, conventional CNNs cannot scale when the input sequences are getting long as the computation complexity increases quadratically along with the receptive field size. Compared to CNNs, the computation complexity of RNNs increases linearly along with the receptive field size. This is because the hidden units of RNNs can only be calculated sequentially. The calculation of each hidden unit depends upon the value of the previous hidden unit. For example, given a sequence with length $L$, RNNs require that all the previous $L-1$ states be calculated to estimate the Lth output. Thus RNNs cannot handle parallel computations efficiently.

To solve the efficiency problem, WaveNet [46] was proposed for modeling raw audio signals and has been used for modeling time sequences in tasks such as speech denoising [37]. WaveNet is an improvement over conventional CNNs, where a "dilated convolution" is applied to reduce the size of filters. A dilated convolution is a convolution with holes. That is, the filters are applied over an area larger than its length by skipping input values with a certain step size. Stacked dilated convolutions enable a network to have a very large receptive field with just a few layers. In [46] a filter length of 2 is applied for modeling the causal audio signals. In this article, following [37], we applied a filter length of 3 to utilise the non-causal information of input sequences. Figure 3 shows, through an example, the WaveNet input-output structure used in this article for both tasks of energy disaggregation and on/off detection.

The architecture of WaveNets for energy disaggregation and on/off detection is shown in Figure 4. The inputs are sequences of aggregate energy readings which are first sent to a 


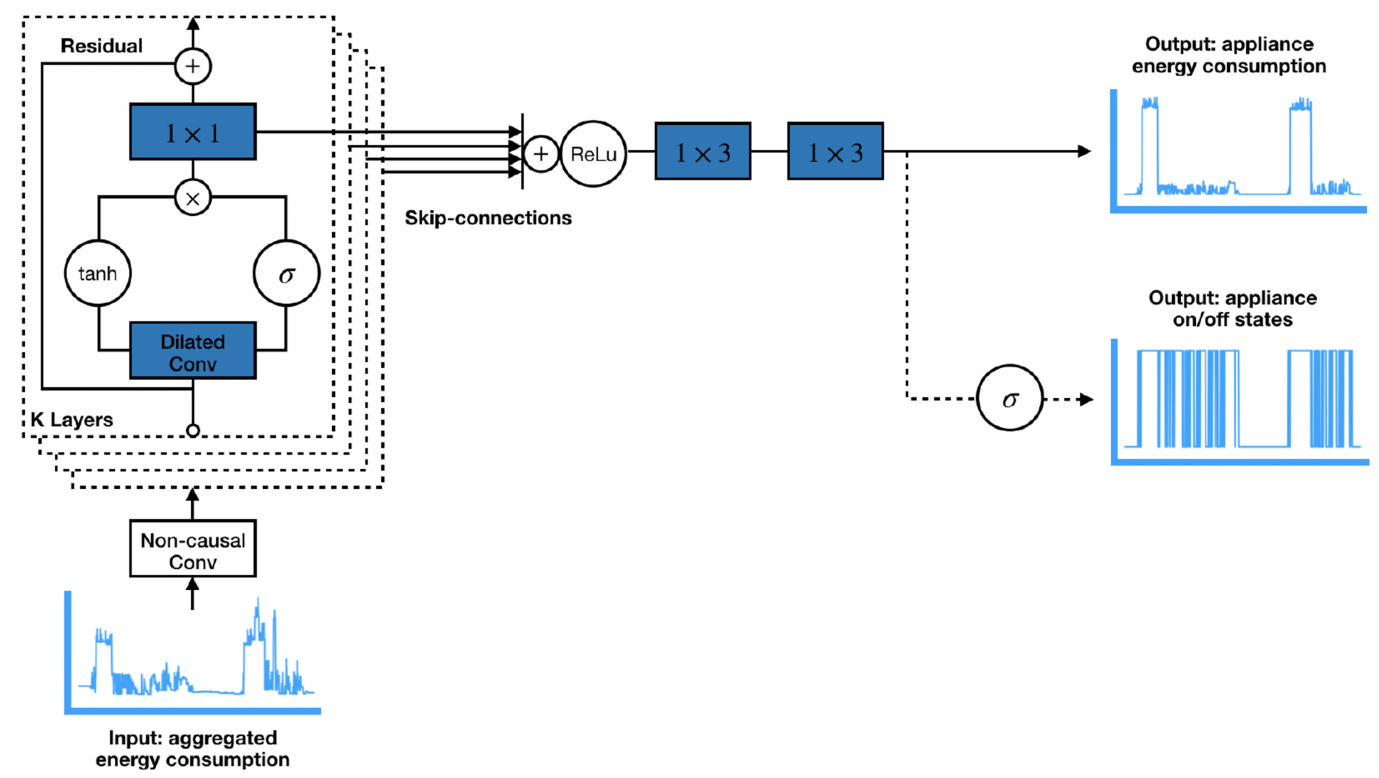

Fig. 4. Architecture of WaveNets for energy disaggregation and on/off detection.

non-causal convolutional layer and then a stack of residual blocks with skipped connections. Following [46], the dilated layers are embedded into the residual blocks. The residual output of each block will be sent to the input of the next block. In each residual block, first a dilated convolutional layer will be applied to the input, the output of which is then respectively sent to a Tanh layer and a Sigmoid layer. Afterwards, the two outputs will be multiplied and followed by a $1 \times 1$ convolutional layer. The skip output of all the residual blocks will be summed and then followed by two $3 \times 1$ convolutional layers, which gives the final output of appliance energy consumption for the task of energy disaggregation. In case of the task of on/off detection, a Sigmoid layer is added to give the final predictions of appliance on/off states.

The following equation characterises the relation between the number of dilated convolutional layers of a WaveNet and its receptive field size:

$$
L=\left(2^{s}-1\right)(m-1)+1,
$$

where $L$ denotes the receptive field size, $s$ denotes the number of dilated convolutional layers and $m$ denotes the filter length applied to each dilated convolutional layer, and in this article, we set $m=3$ following [37]. The number of dilated convolutional layers $s$ used in this article for energy disaggregation and on/off detection will be illustrated in Section 5.4.1.

Since WaveNets do not have recurrent connections [46], they are typically faster than RNNs, especially when applied to long sequences. For a CNN and a WaveNet with the same computational complexity, the receptive field size of the CNN increases linearly with the number of CNN layers, while the receptive field size of the WaveNet increases exponentially with the number of WaveNet layers. That is, with the same receptive field size, a WaveNet has lower computational complexity than a CNN.

\subsection{Training a Model For Energy Disaggregation}

For the task of energy disaggregation, the training of a fast sequence-to-point model based on $\mathrm{CNN} / \mathrm{RNN} /$ WaveNet can be implemented with back-propagation. The inputs are sequences of 
aggregate energy readings $\mathbf{x}$ while the target values are sequences of appliance energy readings $\mathbf{y}$. Assuming an output and the corresponding target value are denoted as $\hat{\mathbf{y}}=$ $\left(\hat{y}_{t+\lfloor L / 2\rfloor}, \ldots, \hat{y}_{t+\lfloor L / 2\rfloor+r-1}\right)$ and $\mathbf{y}=\left(y_{t+\lfloor L / 2\rfloor}, \ldots, y_{t+\lfloor L / 2\rfloor+r-1}\right)$, respectively, the loss can then be calculated using Mean Absolute Error (MAE), which is used as one of the evaluation criteria (see Section 5.3 for details):

$$
\operatorname{loss}(\hat{\mathbf{y}}, \mathbf{y})=\frac{1}{r} \sum_{\tau=\lfloor L / 2\rfloor}^{\lfloor L / 2\rfloor+r-1}\left|\hat{y}_{t+\tau}-y_{t+\tau}\right| .
$$

The loss function is calculated on mini-batch data. When the target field size $r$ equals 1 , Equation (5) degenerates to the conventional sequence-to-point model. After obtaining the loss, the gradient can be calculated and used to update the parameters of the model.

Noted that the models for energy disaggregation of each appliance are trained independently in this article. Thus there is no explicit constraint that the sum of the individual estimates matches the aggregate readings. In this way, the energy disaggregation of one appliance is not constrained by the knowledge of the other appliances used in a household. For example, a CNN model is trained using the aggregate readings as input to infer the energy readings of a single appliance (e.g., kettle). In this case, only kettle is considered as the known appliance while all the other appliances as well as the background noise are regarded as $u_{t}$ in Equation (1) which is expected to be learned by the CNN model.

\subsection{Training a Model for On/off Detection}

Given the aggregated energy consumption, an energy disaggregation model would output the amount of energy an appliance is using at different times. If the on-power threshold of the appliance is known, the output from the energy disaggregation model could be binarised to estimate the on/off states of the appliance. On the other hand, instead of relying on the outputs from energy disaggregation, a binary classifier could be trained for the task by directly using the on/off states of the appliances for training. This article investigates these two learning frameworks, i.e., regression based-learning framework and classification-based learning framework, as shown in Figure 5.

4.4.1 Regression-Based Learning Framework. The regression-based learning framework, as shown in Figure 5(a), tackles the problem of on/off detection by utilising the outputs from energy disaggregation. In concrete, for a given appliance, it first trains a fast sequence-to-point model based on CNN/RNN/WaveNet for energy disaggregation. Thereafter, given a new sequence of aggregate energy readings, the energy readings of the appliance are predicted using the disaggregation model. Finally, it derives the on/off state sequence of the appliance by binarising the predictions according to the on-power threshold of the appliance.

4.4.2 Classification-Based Learning Framework. The classification-based learning framework, as shown in Figure 5(b), tackles the problem of on/off detection by directly training a binary classifier. Concretely, it first binaries the energy readings of a given appliance according to the on-power threshold of the appliance. Thereafter, it trains a binary classifier using the aggregate energy readings as inputs and the binarsied appliance readings as the target values. Finally, given a new sequence of aggregate energy readings, the on/off state sequence of the appliance is predicted using the trained classifier.

To train a fast sequence-to-point binary classifier for a given appliance, the last layer of a $\mathrm{CNN} / \mathrm{RNN} /$ WaveNet is a fully connected layer followed by a sigmoid nonlinearity to represent the probability that the appliance is in the on state. Assuming an output and the corresponding target value are denoted as $\hat{\mathbf{z}}=\left(\hat{z}_{t+\lfloor L / 2\rfloor}, \ldots, \hat{z}_{t+\lfloor L / 2\rfloor+r-1}\right)$ and $\mathbf{z}=\left(z_{t+\lfloor L / 2\rfloor}, \ldots, z_{t+\lfloor L / 2\rfloor+r-1}\right)$ 
(a) Regression based learning framework

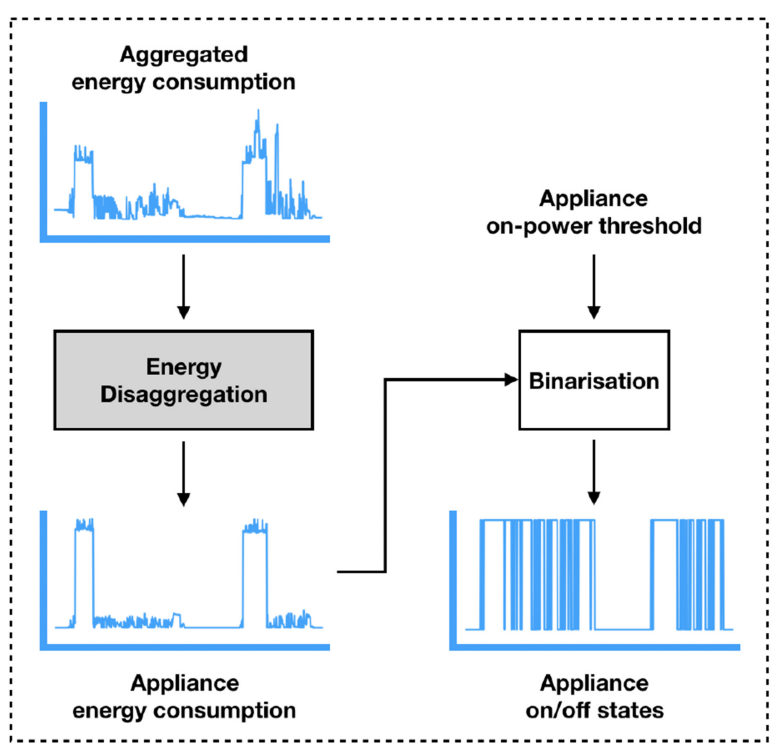

(b) Classification based learning framework

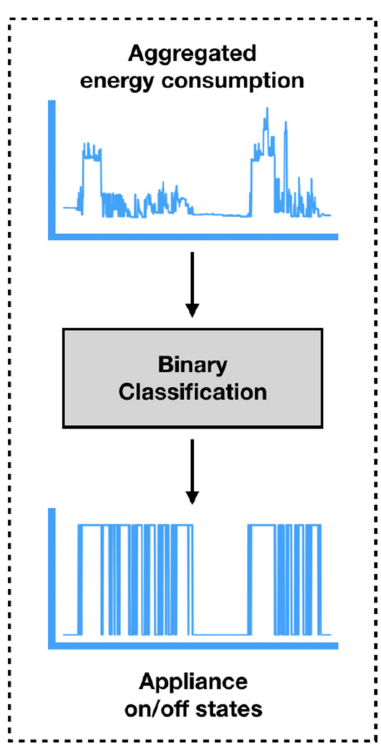

Fig. 5. Regression-based and classification-based learning frameworks.

respectively, the loss can then be calculated using the binary cross-entropy:

$$
\operatorname{loss}(\hat{\mathbf{z}}, \mathbf{z})=-\frac{1}{r} \sum_{\tau=\lfloor L / 2\rfloor}^{\lfloor L / 2\rfloor+r-1}\left(z_{t+\tau} \ln \hat{z}_{t+\tau}+\left(1-z_{t+\tau}\right) \ln \left(1-\hat{z}_{t+\tau}\right)\right) .
$$

Similarly, the loss function is calculated on mini-batch data. When the target filed size $r$ equals 1 , Equation (6) degenerates to the conventional sequence-to-point model. After obtaining the loss, the gradient can be calculated and used to update the parameters of the model.

Similar to energy disaggregation, the models for on/off detection of each appliance are trained independently. That is, when training an on/off detection model for a particular appliance, all the other appliances as well as the background noise are regarded as $u_{t}$ in Equation (1) which is expected to be learned by the on/off detection model.

\section{EXPERIMENTS}

\subsection{Dataset}

The dataset used in this article is REFIT [34], which is a collection of energy consumption data from 20 households in the UK. The readings were recorded around every 8 seconds and covers a period of over 2 years. The dataset contains both house-level energy usage (aggregate readings) and appliance-level energy usage (appliance readings) of more than 10 types of appliances. In this article, we focus on the disaggregation of four types of appliances: kettle, microwave, dish washer and washing machine, which are used by most of the households. The statistics for the energy consumption data of the four types of appliances are described in Table 2.

\subsection{Data Preprocessing}

The REFIT dataset has a mean sampling rate around $7-9$ seconds across the 20 households. Therefore, resampling is required for preparing the training data. To minimise the number of missing 
Table 2. Appliance Data Statistics

\begin{tabular}{c|c|c|c|c}
\hline Appliance & Count & Mean & Standard deviation & Max \\
\hline Kettle & $69,957,575$ & 15.284 & 192.540 & 3,968 \\
Microwave & $69,921,922$ & 5.867 & 86.805 & 3,778 \\
Dishwasher & $71,364,981$ & 34.850 & 259.776 & 3,706 \\
Washing M. & $84,285,754$ & 16.294 & 163.512 & 3,968 \\
\hline
\end{tabular}

Table 3. Households Used for Training and Testing Per Appliance

\begin{tabular}{c|c|c}
\hline Appliance & Training household IDs & Testing household IDs \\
\hline Kettle & {$[2,3,4,5,6,7,8,9,11,12,13]$} & {$[17,19,20,21]$} \\
Microwave & {$[2,3,4,5,6,8,9,10,11,12,15]$} & {$[17,18,19,20]$} \\
Dishwasher & {$[1,2,3,5,6,7,9,10,11,13,15]$} & {$[16,18,20,21]$} \\
Washing M. & {$[1,2,3,5,6,7,8,9,10,11,13,15,16,17]$} & {$[18,19,20,21]$} \\
\hline
\end{tabular}

Table 4. On Power Threshold for Each Appliance in Watts

\begin{tabular}{l|cccc}
\hline & Kettle & Microwave & Dishwasher & Washing M. \\
\hline On power threshold & 2,000 & 200 & 10 & 20 \\
\hline
\end{tabular}

values caused by resampling, we use a resampling rate of 10 seconds, which resulted in 93,976,578 data points. Secondly, following [23], we filled the gaps in the data shorter than 3 minutes by forward-filling assuming that the gaps are caused by RF issues and filled the gaps longer than 3 minutes with zeros assuming that the gaps are caused by the appliance being switched off. Thirdly, for each type of appliance and the aggregate, we normalised the data by subtracting the mean values and dividing by the corresponding standard deviations.

Thereafter, for each household, we extracted all the possible segments of length $(L+r-1)$ from the aggregate readings by a sliding window of step-size $r$, where $L$ indicates the size of the receptive field and $r$ indicates the size of the target field. These segments of aggregate readings are used as inputs for training and testing. For each of the aggregate segments, we obtained the corresponding target sequence by extracting a segment of consecutive appliance readings of length $r$ such that the center of the two segments are aligned. Moreover, we remove any input sequence and its corresponding target sequence where the target sequence contains an appliance reading that is larger than the corresponding aggregate reading in the input sequence. Since not every household has all the four appliances, we used the data from the last four households for testing and the data from the rest of the households for training, as shown in Table 3. It has to be noted that this article aims at investigating the model performance on unseen households. Therefore the data from different sets of households are used for training and testing.

As for on/off detection, instead of normalising the appliance readings, we obtain the output data by binarising the appliance readings using the on power thresholds shown in Table 4 in accordance with the previous studies [49] and [23].

\subsection{Evaluation Metrics}

For the task of energy disaggregation, we used two metrics for evaluation in this article, i.e., MAE and normalised Signal Aggregate Error (SAE). MAE is a measurement of errors that averages 
over the differences between all the predictions with respect to the real consumptions, which is less sensitive with outliers. SAE is a measurement of errors that sums all the differences between the predictions and the real consumptions over a period of time, e.g., a day, a week, and a month. In our case, the evaluation is over the whole time period of the testing households' data collection. The formal definitions of MAE and SAE are as follows:

$$
M A E=\frac{1}{T} \sum_{t=1}^{T}\left|\hat{y}_{t}-y_{t}\right|,
$$

where $\hat{y}_{t}$ indicates the prediction of an appliance's energy usage at time $t$ and $y_{t}$ indicates the corresponding ground truth.

$$
S A E=\frac{|\hat{a}-a|}{a},
$$

where $\hat{a}=\sum_{t} \hat{y}_{t}$ and $a=\sum_{t} y_{t}$, respectively, indicate the predicted energy consumption of an appliance over a certain time period and the corresponding ground truth.

For the task of on/off detection, we used F1 score to evaluate the performance of different models as the dataset is extremely imbalanced. For example, kettle is on only for about $1 \%$ of the time. F1 score [21] can be interpreted as a harmonic average of the precision and recall:

$$
F 1=2 \times \frac{\text { precision } \times \text { recall }}{\text { precision }+ \text { recall }},
$$

where precision is the fraction of true positive instances among the predicted positive instances, while recall is the fraction of true positive instances over the total number of positive instances.

\subsection{Experimental Results For Energy Disaggregation}

5.4.1 Experiment Setup. For energy disaggregation, we trained three groups of neural network models for each of the four types of appliances as shown in Table 2 independently. That is, a model trained for energy disaggregation of a particular appliance (e.g., kettle) only assumes the existence of that appliance (kettle) while treating all the other appliances as unknown. The first group is based on our implementation of the 5-layer CNN proposed in [49]. The second group is based on our implementation of the two-layer bidirectional RNN with LSTM units (indicated by BLSTM) proposed in [23]. The third group is based on a three-layer bidirectional RNN with GRUs (indicated by BGRU). The fourth group is based on the WaveNet as shown in Section 4.2. We use the Adam optimizer [27] with a learning rate of 0.001 to minimise the loss as shown in Equation (5). These hyper-parameters are chosen experimentally. We used TitanX with 12 GB GPU memory to train all the energy disaggregation and on/off detection models. Moreover, to compare with the traditional machine learning approaches, we also trained a group of FHMMs following the implementation in the NILMTK Toolkit [6].

For each group of neural network models, we explored the influence of two parameters. The first parameter is the receptive field size $L$. In this article, we used input sequences with a range of receptive field sizes $15,31,63,127,255,511,1,023,2,047$, which corresponds to numbers of layers 3 , 4, 5, 6, 7, 8, 9, 10 in WaveNet models. Note that the receptive field size of 1,023 and 2,047 were not applied to the $\mathrm{CNN}$ models for the sake of computation cost in training. The second parameter is the target field size $r$ for which we experimented with four different values $1,10,100$ and 1,000. We used a mini-batch size of 128 for training all the models.

For most appliances, the duration that an appliance is being used is much smaller than it is not, i.e., the readings are extremely imbalanced between those representing the appliance is in use and those representing it is not. For example, the readings that are less than 10 watts is around $99 \%$ for kettle. In such cases, a model that always predicts a very small value, e.g., zero, may perform 
Table 5. The Appliance-level MAE in Unit of Watt and SAE

\begin{tabular}{l|c|ccccc}
\hline Metrics & Methods & Kettle & Microwave & Dishwasher & Washing M. & Overall \\
\hline \multirow{6}{*}{ MAE } & Always-zero & 10.157 & 4.386 & 20.784 & 6.189 & $10.378 \pm 6.359$ \\
& FHMM [6] & 89.911 & 208.869 & 62.463 & 79.395 & $110.159 \pm 57.825$ \\
& CNN [49] & 5.454 & 4.002 & 21.014 & 4.970 & $8.860 \pm 7.036$ \\
& BLSTM [23] & 4.939 & 3.903 & 13.854 & 3.189 & $6.471 \pm 4.307$ \\
& BGRU & 4.839 & 3.696 & 12.110 & 3.602 & $6.061 \pm 3.525$ \\
& WaveNet & $\mathbf{4 . 7 2 6}$ & $\mathbf{3 . 6 8 6}$ & $\mathbf{1 0 . 2 9 6}$ & $\mathbf{3 . 0 8 0}$ & $\mathbf{5 . 4 4 6} \pm 2.860$ \\
\hline \multirow{5}{*}{ SAE } & Always-mean & 1.347 & 0.713 & 1.121 & 2.121 & $1.325 \pm 0.512$ \\
& FHMM [6] & 4.374 & 14.583 & 1.603 & 3.278 & $5.959 \pm 5.075$ \\
& CNN [49] & 0.258 & 0.797 & 0.976 & 0.440 & $0.617 \pm 0.283$ \\
& BLSTM [23] & 0.296 & $\mathbf{0 . 5 5 7}$ & 0.400 & 0.252 & $0.376 \pm 0.117$ \\
& BGRU & 0.249 & 0.644 & 0.290 & $\mathbf{0 . 2 0 8}$ & $0.347 \pm 0.173$ \\
& WaveNet & $\mathbf{0 . 2 2 4}$ & 0.666 & $\mathbf{0 . 1 9 2}$ & 0.267 & $\mathbf{0 . 3 3 7 \pm 0 . 1 9 1}$ \\
\hline
\end{tabular}

Best results are shown in bold.

well in terms of MAE. Therefore, we employ a naive baseline model, i.e., always predicting zero (always-zero). The metric SAE focuses on the total energy consumption over a period of time, which makes the mean value of an appliance's energy consumption a promising prediction. To this end, we employ another naive baseline model, i.e., always predicting the mean value (alwaysmean).

5.4.2 Result Analysis. Table 5 shows the best MAE together with the corresponding SAE achieved by the models within each group with a fixed target field size of 100 . We can see that the WaveNet model achieves the best MAE over all the four appliances. In particular for dishwasher and washing machine, the WaveNet model reduces the MAE by $51 \%$ and $38 \%$ comparing to the CNN model while by $14 \%$ and $3 \%$ comparing to the best results from the RNN models (BLSTM and BGRU). As for kettle and microwave, the WaveNet model and the BGRU model obtain similar MAEs. In the case of SAE, the WaveNet model achieves better results for kettle and dishwasher while the RNN models achieve better results for microwave and washing machine. Overall, in terms of the mean and standard deviation of the MAE and SAE derived from the results of the disaggregated sequences of all the four appliances, the neural network models have much better performance than the FHMMs, and the WaveNet model outperforms the other three types of neural network models as well as the two naive baselines.

Among the four appliances, microwave is the only one that all the three neural network models achieve comparable results as that of the model of always-zero and always-mean. A closer inspection of the REFIT dataset shows that microwaves were mostly operated on either the off mode or the standby mode ( $0-5$ watts) and the latter composes of more than $99.6 \%$ of the readings which is the highest among the four appliances.

To have a visual understanding of the disaggregation results, Figure 6 shows for each type of appliance an excerpt of the predictions together with the target values with respect to the CNN, BLSTM, BGRU and WaveNet models that achieve the best MAE (as shown in Table 5). It can be seen that the disaggregation results for kettle are similar among the four models. As for microwave, the WaveNet model has some predictions that are larger than the target values while the predictions of the BGRU model are mostly smaller than the target values. The CNN and LSTM models however do not recognise the operation of the microwave, which is in line with the fact that the corresponding MAE of the CNN and BLSTM models are similar to that of the always-zero model. As for dishwasher and washing machine, the predictions of the WaveNet model are finer and closer to the target values 

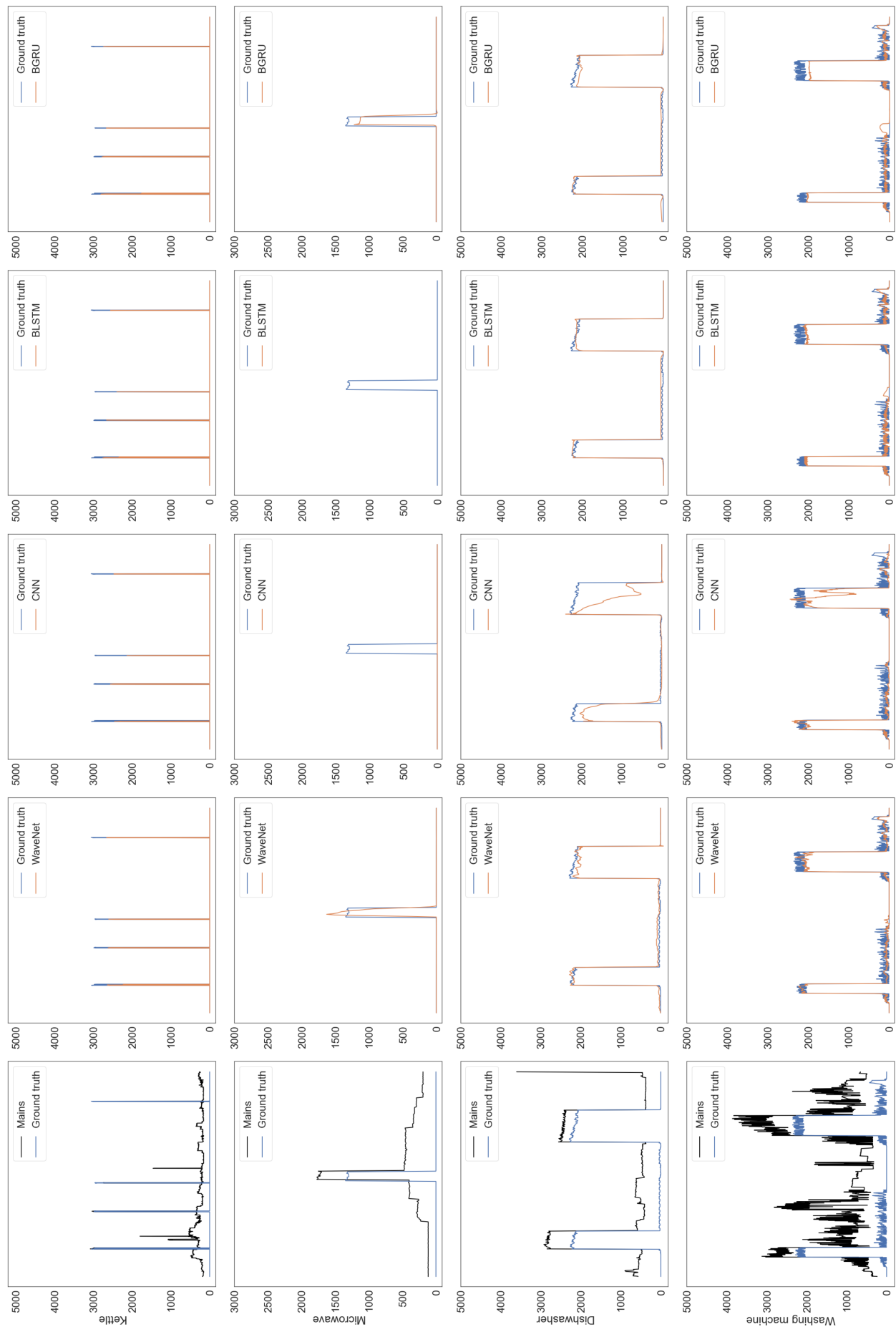

Fig. 6. Excerpts of disaggregation results with respect to the CNN, BLSTM, BGRU and WaveNet model that achieved the best MAE. 

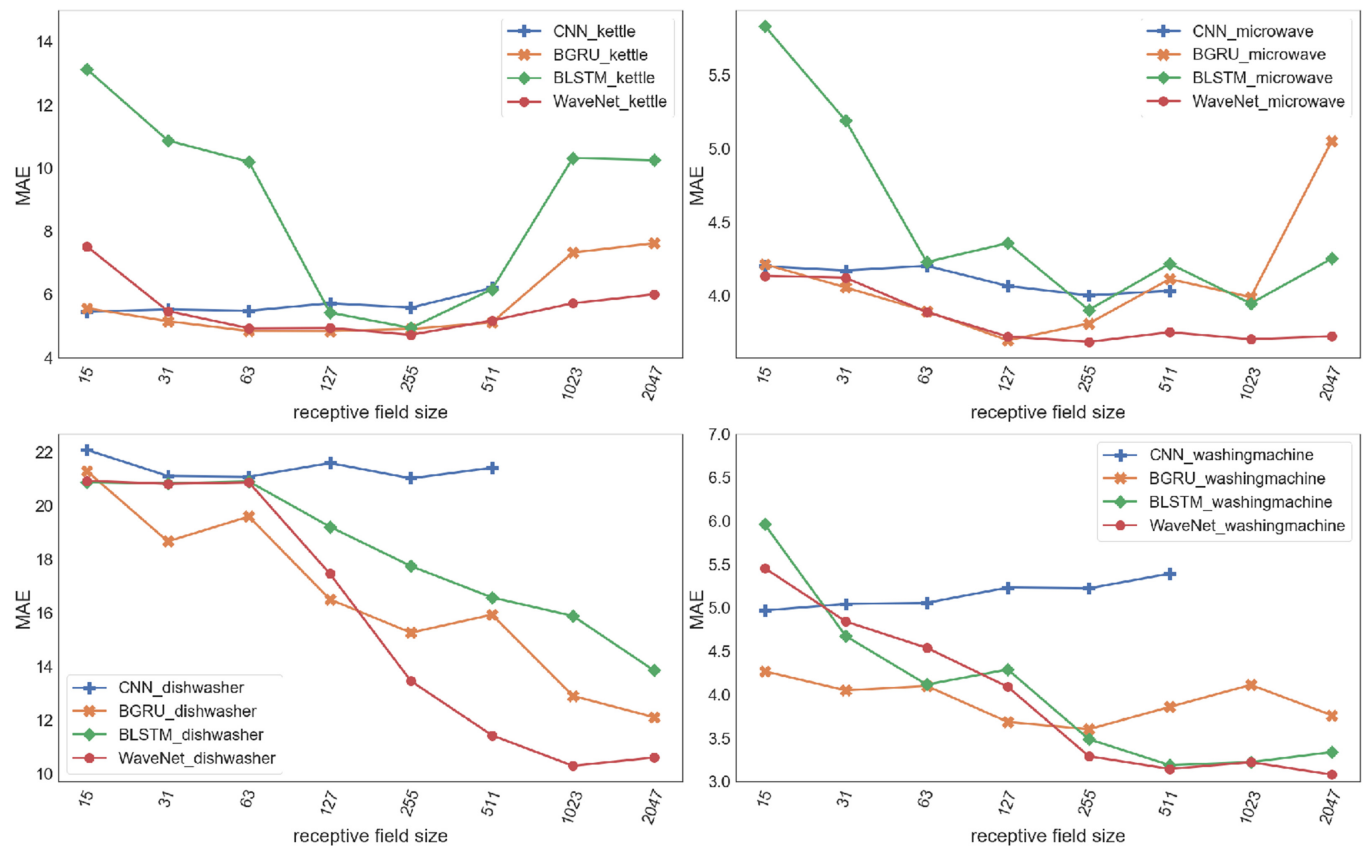

Fig. 7. MAE of the CNN, BLSTM, BGRU and WaveNet models with different receptive field sizes for the four appliances.

compare to that of the RNN models, and the predictions of the CNN model are much noisier than the other two models.

To investigate how the length of input sequences influences the model performance, we compare the MAEs achieved by the CNN, BLSTM, BGRU and WaveNet models as shown in Figure 7. Note that the receptive field size of 1023 and 2047 were only applied to the WaveNet and RNN models for the sake of computation cost. The size of the receptive field in general does not have much influence on the performance of the CNN models compared to the other two groups of models. The RNN models in general achieve better MAE when the size of the receptive field gets longer but when the receptive filed size is larger than 255 the performance gets worse. As for the WaveNet models, there is a clear tendency that its performance is getting better with longer receptive fields in the cases of dishwasher and washing machine. An explanation is that dishwashers and washing machines have relatively longer period of operation and the models need more information to capture the energy consumption patterns. In the case of kettle, the WaveNet models achieve better MAE with the size of the receptive field getting longer up to 255 and thereafter the performance starts getting worse. This may be explained by the fact that kettles usually have a short operation time and any longer receptive field will introduce too much noise.

Training efficiency is also an important factor when comparing models. Figure 8 shows the training time per mini-batch needed by the CNN, BLSTM, BGRU and WaveNet models. We can see that when the receptive field size is above 511 the computation time of the CNN models increases quadratically. The WaveNet models have the lowest computation cost when the receptive field size becomes substantially large $(\geq 511)$ among the three groups of models. Furthermore, the WaveNet models converge much quicker than the other three groups of models. For example, for washing machine, the number of iterations that the $\mathrm{CNN}$ models and the RNN models needed for training until convergence is more than 3 to 4 times of that needed by the WaveNet models. 


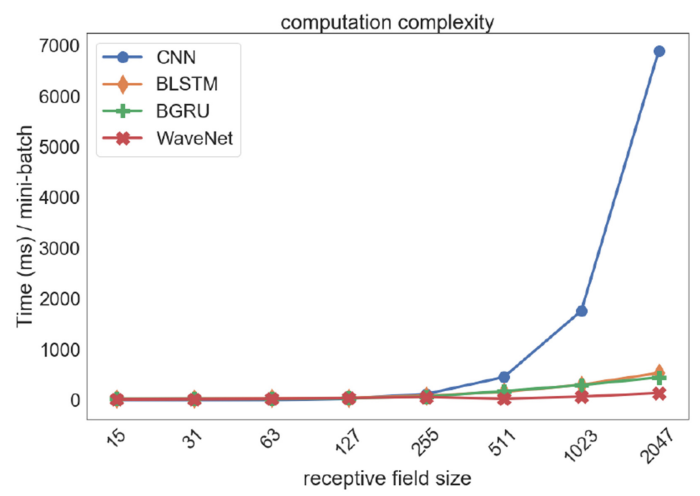

Fig. 8. Computation time per iteration for the CNN, BLSTM, BGRU and WaveNet models with different receptive field sizes.

Table 6. MAE of the WaveNet Models with Different Target Field Sizes

\begin{tabular}{c|c|cccc}
\hline Model & Target field size & Kettle & Microwave & Dishwasher & Washing M. \\
\hline $\begin{array}{c}\text { WaveNet } \\
\text { (receptive field size=127) }\end{array}$ & 1 & 10.279 & 4.294 & 20.832 & 5.726 \\
& 10 & & & & \\
& 100 & $\mathbf{5 . 0 6 4}$ & 3.926 & $\mathbf{1 7 . 6 6 3}$ & 4.296 \\
& 1000 & 5.446 & $\mathbf{3 . 7 9 4}$ & 18.142 & $\mathbf{3 . 9 0 6}$ \\
\hline
\end{tabular}

The best result per appliance is shown in bold.

Target field size is a parameter that is worth exploring as well. In Table 6 we show the relation between the target field size and the performance of the WaveNet models in terms of MAE with a fixed receptive field size of 127 . We can see that for all the four appliances there is a tendency that the longer target fields achieve better MAE. This is because the longer target fields provide more training samples per mini-batch which is similar to the effect of applying a larger batch size and is more likely to converge to global optima. Comparing to using a larger batch size, the computation efficiency of using longer target fields is much higher due to shared computations.

\subsection{Experimental Results For On/off Detection}

5.5.1 Experiment Setup. As for the task of on/off detection, our aim is to compare the performance of the regression based learning framework and the classification based learning framework as proposed in Section 4.4. As it has been shown in the previous subsection that WaveNets and BGRUs achieve better performance for the task of energy disaggregation, we trained two groups of WaveNet models and BGRU models for each of the four types of appliances as shown in Table 2 following the two learning frameworks. Similar to energy disaggregation, a model trained for on/off detection of a particular appliance (e.g., kettle) only assumes the existence of that appliance (kettle) while treating all the other appliances as unknown. We experimented with a range of receptive field sizes $15,31,63,127,255,511,1023,2047$. The Adam optimizer is used with a learning rate of 0.001 to minimise the loss function shown in Equation (5) for the regression based learning framework and in Equation (6) for the classification based learning framework.

5.5.2 Result Analysis. Figure 9 shows the F1 scores obtained by the WaveNet and BGRU models trained respectively under the two learning frameworks with an increasing receptive field size. For the binary classifier under the classification based learning framework, we use a cut-off probability 

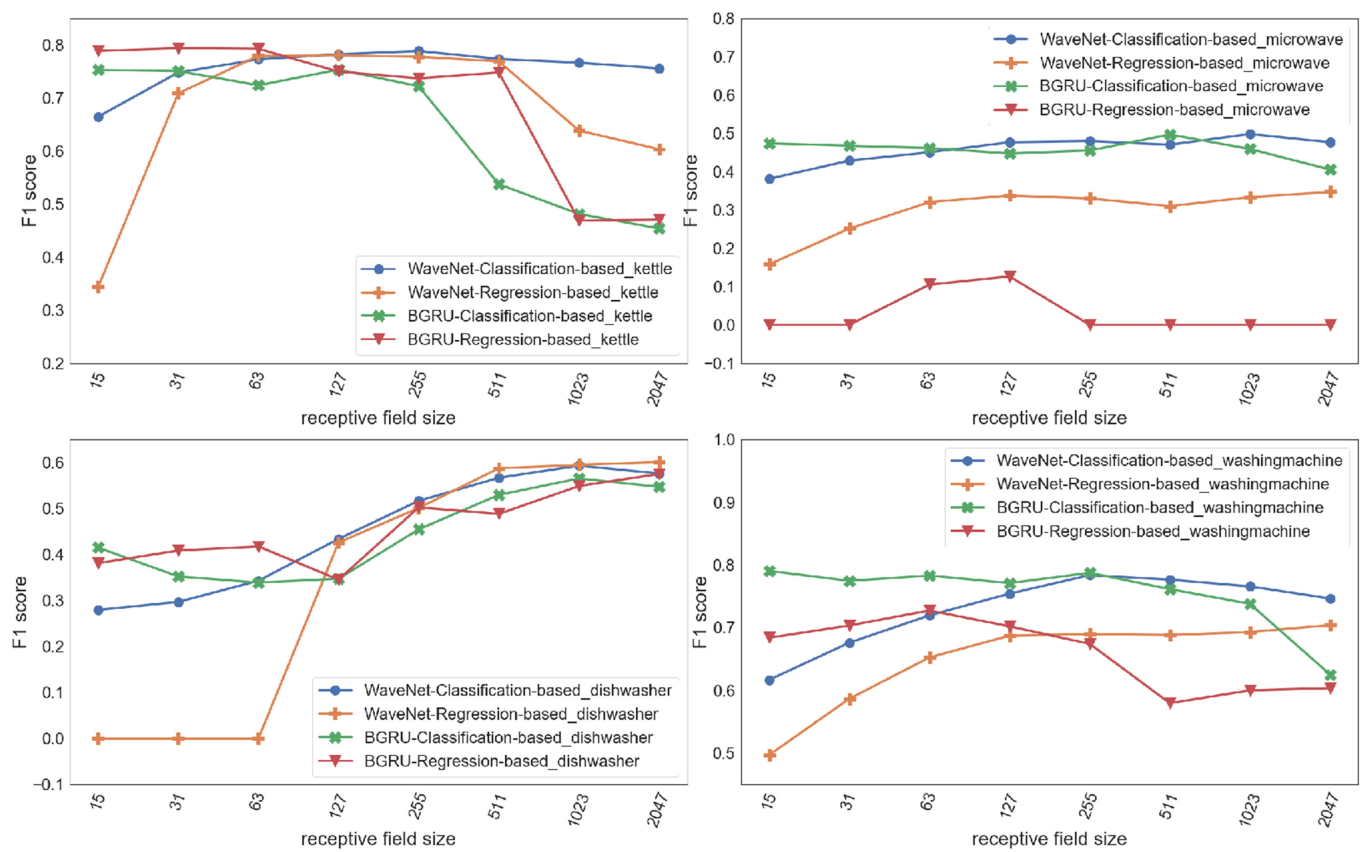

Fig. 9. F1 score of the WaveNet and BGRU models trained following the two learning frameworks.

of 0.3 , that is, when the classifier outputs a value larger than 0.3 we consider the appliance is in the on state otherwise in the off state. This cut-off value is chosen empirically following the previous work on sound event detection [48]. We leave the exploration of the threshold hyper-parameter for future work.

We can see that in the case of microwave and washing machine, the classification-based learning framework achieves better F1 score than the regression-based learning framework for both the WaveNet and BGRU models, while the performance of the WaveNet models and the BGRU models under the classification based learning framework are similar. As for kettle and dishwasher, the two learning frameworks achieve similar performance in terms of the best F1 score achieved by the WaveNet models. The BGRU models in general achieve better performance under the regression based learning framework in the case of kettle while the performance is similar in the case of dishwasher.

\section{CONCLUSIONS}

In this article, we investigated the problem of energy disaggregation together with the problem of appliance on/off detection. Firstly, we formalised both problems and illustrated the learning/ training paradigms used in the literature, which motivated us to introduce the fast-sequence-topoint learning paradigm. By comparing with CNN models and RNN models, we studied the application of the recently proposed WaveNet models to the problem of energy disaggregation. With an evaluation on a real-world dataset, we showed that our disaggregation models based on WaveNets outperform the previous works based on CNNs and RNNs. The empirical evidence demonstrates WaveNets' superiority in handling long sequences. By an extensive experiment with input sequences of varying receptive field sizes, we have shown how the receptive field size affects the disaggregation performance for different appliances. Furthermore, we studied the problem of appliance on/off detection as a natural continuation of the disaggregation problem and investigated 
the performance of two learning frameworks: (1) a regression-based learning framework utilising the results from energy disaggregation; and (2) a classification-based learning framework that directly trains a binary classifier. We showed empirically that the classification-based learning framework outperforms the regression-based learning framework in terms of F1 score. This indicates that for applications targeting at appliance on/off states, directly training a binary classifier would be a better choice.

For future work, we intend to explore the use of prior knowledge to enhance the learning of WaveNet models. Another interesting direction for future work is to investigate multimodal learning and multi-task learning techniques (e.g., $[1,2]$ ) to improve the results of energy disaggregation and on/off detection. For example, with multimodal learning, we could relate the on/off states of an appliance to the amount of energy the appliance consumes. As for multi-task learning, we could perform the task of energy disaggregation and the task of on/off detection simultaneously.

\section{REFERENCES}

[1] Giuseppe Aceto, Domenico Ciuonzo, Antonio Montieri, and Antonio Pescapé. 2019. MIMETIC: Mobile encrypted traffic classification using multimodal deep learning. Computer Networks 165 (2019), 106944. DOI : https://doi.org/10. 1016/j.comnet.2019.106944

[2] Giuseppe Aceto, Domenico Ciuonzo, Antonio Montieri, and Antonio Pescapé. 2020. Toward effective mobile encrypted traffic classification through deep learning. Neurocomputing 409 (2020), 306-315. DOI : https://doi.org/10.1016/ j.neucom.2020.05.036

[3] José Alcalá, Oliver Parson, and Alex Rogers. 2015. Detecting anomalies in activities of daily living of elderly residents via energy disaggregation and cox processes. In Proceedings of the 2nd ACM International Conference on Embedded Systems for Energy-Efficient Built Environments. ACM, 225-234.

[4] K. Basu, V. Debusschere, S. Bacha, U. Maulik, and S. Bondyopadhyay. 2015. Nonintrusive load monitoring: A temporal multilabel classification approach. IEEE Transactions on Industrial Informatics 11, 1 (2015), 262-270.

[5] Kaustav Basu, Vincent Debusschere, and Bacha Seddik. 2012. Load identification from power recordings at meter panel in residential households. Proceedings of the 2012 20th International Conference on Electrical Machines (ICEM '12). 2098-2104. DOI : https://doi.org/10.1109/ICElMach.2012.6350172

[6] Nipun Batra, Jack Kelly, Oliver Parson, Haimonti Dutta, William Knottenbelt, Alex Rogers, Amarjeet Singh, and Mani Srivastava. 2014. NILMTK: An open source toolkit for non-intrusive load monitoring. In Proceedings of the 5th International Conference on Future Energy Systems.

[7] M. Z. A. Bhotto, S. Makonin, and I. V. Bajic. 2017. Load disaggregation based on aided linear integer programming. IEEE Transactions on Circuits and Systems II: Express Briefs 64, 7 (2017), 792-796.

[8] Kunjin Chen, Qin Wang, Ziyu He, Kunlong Chen, Jun Hu, and Jinliang He. 2018. Convolutional sequence to sequence non-intrusive load monitoring. The fournal of Engineering 17 (2018), 1860-1864.

[9] Junyoung Chung, Caglar Gulcehre, Kyunghyun Cho, and Yoshua Bengio. 2014. Empirical evaluation of gated recurrent neural networks on sequence modeling. In Proceedings of the NIPS 2014 Workshop on Deep Learning and Representation Learning.

[10] Yann N. Dauphin, Angela Fan, Michael Auli, and David Grangier. 2017. Language modeling with gated convolutional networks. In Proceedings of the 34th International Conference on Machine Learning. JMLR.org, 933-941.

[11] Dominik Egarter, Anita Sobe, and Wilfried Elmenreich. 2013. Evolving non-intrusive load monitoring. In Proceedings of the International Conference on the Applications of Evolutionary Computation. Vol. 7835. 182-191.

[12] Ehsan Elhamifar and Shankar Sastry. 2015. Energy disaggregation via learning powerlets and sparse coding. In Proceedings of the 29th AAAI Conference on Artificial Intelligence. AAAI Press, 629-635.

[13] Corinna Fischer. 2008. Feedback on household electricity consumption: A tool for saving energy?Energy Efficiency 1 , 1 (2008), 79-104.

[14] Jon Froehlich. 2009. Promoting energy efficient behaviors in the home through feedback: The role of human computer interaction. In Proceedings of the HCIC 2009 Winter Workshop.

[15] Jon Froehlich, Eric Larson, Sidhant Gupta, Gabe Cohn, Matthew Reynolds, and Shwetak Patel. 2011. Disaggregated end-use energy sensing for the smart grid. IEEE Pervasive Computing 10, 1 (2011), 28-39.

[16] Alex Graves, Abdel-rahman Mohamed, and Geoffrey Hinton. 2013. Speech recognition with deep recurrent neural networks. In Proceedings of the IEEE International Conference on Acoustics, Speech and Signal Processing. 6645-6649.

[17] Alon Harell, Stephen Makonin, and Ivan V. Bajić. 2019. WaveNILM: A causal neural network for power disaggregation from the omplex power signal. In Proceedings of the IEEE International Conference on Acoustics, Speech and Signal Processing. 8335-8339. 
[18] George W. Hart. 1992. Nonintrusive appliance load monitoring. Proceedings of the IEEE 80, 12 (1992), 1870-1891.

[19] K. He, L. Stankovic, J. Liao, and V. Stankovic. 2018. Non-intrusive load disaggregation using graph signal processing. IEEE Transactions on Smart Grid 9, 3 (2018), 1739-1747.

[20] Sepp Hochreiter and Jürgen Schmidhuber. 1997. Long short-term memory. Neural Computation 9, 8 (1997), 1735-1780.

[21] László A. Jeni, Jeffrey F. Cohn, and Fernando De La Torre. 2013. Facing imbalanced data-recommendations for the use of performance metrics. In Proceedings of the 2013 Humaine Association Conference on Affective Computing and Intelligent Interaction. IEEE Computer Society, 245-251.

[22] Jie Jiang, Mark Hoogendoorn, Diederik Roijers, Qiuqiang Kong, and Nigel Gilbert. 2018. Predicting appliance usage status in home like environments. In Proceedings of the 23rd International Conference on Digital Signal Processing.

[23] Jack Kelly and William Knottenbelt. 2015. Neural NILM: Deep neural networks applied to energy disaggregation. In Proceedings of the 2nd ACM International Conference on Embedded Systems for Energy-Efficient Built Environments. ACM, 55-64.

[24] Jack Kelly and William Knottenbelt. 2015. The UK-DALE dataset, domestic appliance-level electricity demand and whole-house demand from five UK homes. Scientific Data 2, 150007 (2015), 1-14.

[25] M. Khodayar, J. Wang, and Z. Wang. 2019. Energy disaggregation via deep temporal dictionary learning. IEEE Transactions on Neural Networks and Learning Systems 31, 5 (2019), 1-14.

[26] Hyungsul Kim, Manish Marwah, Martin F. Arlitt, Geoff Lyon, and Jiawei Han. 2011. Unsupervised disaggregation of low frequency power measurements. In Proceedings of the 2011 SIAM International Conference on Data Mining. 747-758.

[27] Diederik P. Kingma and Jimmy Ba. 2014. Adam: A method for stochastic optimization. In Proceedings of the 3rd International Conference on Learning Representations.

[28] J. Zico Kolter, Siddarth Batra, and Andrew Y. Ng. 2010. Energy disaggregation via discriminative sparse coding. In Proceedings of the 23rd International Conference on Neural Information Processing Systems - Volume 1 (NIPS '10). Curran Associates Inc., Red Hook, NY, 1153-1161.

[29] J. Zico Kolter and Tommi Jaakkola. 2012. Approximate inference in additive factorial HMMs with application to energy disaggregation. In Proceedings of the 15th International Conference on Artificial Intelligence and Statistics. 1472 1482.

[30] Kosuke Suzuki, Shinkichi Inagaki, Tatsuya Suzuki, Hisahide Nakamura, and Koichi Ito. 2008. Nonintrusive appliance load monitoring based on integer programming. In Proceedings of the 2008 SICE Annual Conference. 2742-2747.

[31] Alex Krizhevsky, Ilya Sutskever, and Geoffrey E. Hinton. 2017. ImageNet classification with deep convolutional neural networks. Communications of the ACM 60, 6 (May 2017), 84-90.

[32] D. Li and S. Dick. 2019. Residential household non-intrusive load monitoring via graph-based multi-label semisupervised learning. IEEE Transactions on Smart Grid 10, 4 (2019), 4615-4627.

[33] Lukas Mauch and Bin Yang. 2015. A new approach for supervised power disaggregation by using a deep recurrent LSTM network. In Proceedings of the 2015 IEEE Global Conference on Signal and Information Processing. 63-67.

[34] David Murray, Lina Stankovic, and Vladimir Stankovic. 2017. An electrical load measurements dataset of United Kingdom households from a two-year longitudinal study. Scientific Data 4, 160122 (2017), 1-12.

[35] Oliver Parson, Siddhartha Ghosh, Mark Weal, and Alex Rogers. 2012. Non-intrusive load monitoring using prior models of general appliance types. In Proceedings of the 26th AAAI Conference on Artificial Intelligence. AAAI Press, 356-362.

[36] Razvan Pascanu, Tomas Mikolov, and Yoshua Bengio. 2013. On the difficulty of training recurrent neural networks. In Proceedings of the International Conference on Machine Learning. 1310-1318.

[37] Dario Rethage, Jordi Pons, and Xavier Serra. 2018. A WaveNet for speech denoising. In Proceedings of the 2018 IEEE International Conference on Acoustics, Speech and Signal Processing. 5069-5073.

[38] Kiarash Shaloudegi, András György, Csaba Szepesvári, and Wilsun Xu. 2016. SDP relaxation with randomized rounding for energy disaggregation. In Proceedings of the 30th International Conference on Neural Information Processing Systems. 4985-4993.

[39] Changho Shin, Sunghwan Joo, Jaeryun Yim, Hyoseop Lee, Taesup Moon, , and Wonjong Rhee. 2019. Subtask gated networks for non-intrusive load monitoring. In Proceedings of 33rd AAAI Conference on Artificial Intelligence. 11501157.

[40] Olivier Sidler. 2003. DSM: Major findings of an end-use metering campaign in 400 households of four European countries. In ECEEE Summer Study Proceedings. 467-474.

[41] O. Sidler and P. Waide. 1999. Metering matters! Appliance Efficiency 3, 4 (1999).

[42] S. Singh and A. Majumdar. 2018. Deep sparse coding for non-intrusive load monitoring. IEEE Transactions on Smart Grid 9, 5 (2018), 4669-4678.

[43] V. Singhal, J. Maggu, and A. Majumdar. 2019. Simultaneous detection of multiple appliances from smart-meter measurements via multi-label consistent deep dictionary learning and deep transform learning. IEEE Transactions on Smart Grid 10, 3 (2019), 2969-2978. 
[44] Tharmakulasingam Sirojan, B. Toan Phung, and Eliathamby Ambikairajah. 2018. Deep neural network based energy disaggregation. In Proceedings of the 2018 IEEE International Conference on Smart Energy Grid Engineering. 73-77.

[45] S. M. Tabatabaei, S. Dick, and W. Xu. 2017. Toward non-intrusive load monitoring via multi-label classification. IEEE Transactions on Smart Grid 8, 1 (2017), 26-40.

[46] Aäron van den Oord, Sander Dieleman, Heiga Zen, Karen Simonyan, Oriol Vinyals, Alex Graves, Nal Kalchbrenner, Andrew W. Senior, and Koray Kavukcuoglu. 2016. WaveNet: A generative model for raw audio. arXiv preprint arXiv:1609.03499(2016).

[47] S. Verma, S. Singh, and A. Majumdar. 2019. Multi label restricted Boltzmann machine for non-intrusive load monitoring. In Proceedings of the 2019 IEEE International Conference on Acoustics, Speech and Signal Processing (ICASSP'19). 8345-8349.

[48] Y. Xu, Q. Kong, W. Wang, and M. D. Plumbley. 2018. Large-scale weakly supervised audio classification using gated convolutional neural network. In Proceedings of the 2018 IEEE International Conference on Acoustics, Speech and Signal Processing (ICASSP'18). 121-125.

[49] Chaoyun Zhang, Mingjun Zhong, Zongzuo Wang, Nigel Goddard, and Charles Sutton. 2018. Sequence-to-point learning with neural networks for nonintrusive load monitoring. In Proceedings of the 32nd AAAI Conference on Artificial Intelligence. 2604-2611.

[50] B. Zhao, K. He, L. Stankovic, and V. Stankovic. 2018. Improving event-based non-intrusive load monitoring using graph signal processing. IEEE Access 6 (2018), 53944-53959.

[51] B. Zhao, L. Stankovic, and V. Stankovic. 2016. On a training-less solution for non-intrusive appliance load monitoring using graph signal processing. IEEE Access 4 (2016), 1784-1799.

[52] Mingjun Zhong, Nigel Goddard, and Charles Sutton. 2014. Signal aggregate constraints in additive factorial HMMs, with application to energy disaggregation. In Proceedings of the Advances in Neural Information Processing Systems. $3590-3598$.

Received August 2019; revised September 2020; accepted December 2021 